\title{
Efeito da Seleção para Crescimento na Permanência de Vacas Nelore no Rebanho até Cinco Anos de Idade ${ }^{1}$
}

\section{Maria Eugênia Zerlotti Mercadante ${ }^{2}$, Alexander George Razook 2,3 , Joslaine Noely dos Santos Gonçalves Cyrillo ${ }^{2,3}$, Leopoldo Andrade de Figueiredo ${ }^{2}$}

\begin{abstract}
RESUMO - Registros de 1021 vacas Nelore foram analisados com o objetivo de estudar o efeito da seleção para crescimento na permanência de fêmeas no rebanho até cinco anos de idade, considerando-se que ela foi selecionada (P5|S). A variável, codificada como $0=$ descartada ou $1=$ não descartada, foi analisada com modelo de limiar. As vacas, nascidas de 1981 a 1997 , foram provenientes dos rebanhos selecionados ( $\mathrm{NeS}$ e NeT) e controle (NeC) da Estação Experimental de Zootecnia de Sertãozinho. Nos rebanhos NeS e NeT, foram selecionados animais com diferenciais de seleção máximos, dentro de rebanho e ano, e em NeC aqueles com diferenciais de seleção nulos, para peso ao sobreano. Após 3,5 gerações de seleção, o peso à seleção das fêmeas dos rebanhos $\mathrm{NeS}$ e NeT foi $22 \%$ maior que das fêmeas do NeC. A média observada de P5|S foi 68\%. Os efeitos de rebanho e peso à seleção foram não significativos. O efeito da geração ao qual a fêmea pertence foi significativo, com tendência, similar nos três rebanhos, de diminuição da P5|S ao longo dos anos, refletindo as mudanças de manejo de novilhas à primeira monta, e não o efeito da seleção para crescimento. As variâncias de origem genética aditiva, estimadas por modelo animal, modelo touro e modelo touro avô-materno, foram 0,$06 ; 0,16$ e 0,17 , proporcionalmente à $\sigma^{2}$ fixa em uma unidade da medida, e as herdabilidades iguais a 0,06 $\pm 0,06 ; 0,15 \pm 0,13$ e $0,16 \pm 13$, respectivamente. As médias dos valores genéticos dos touros, por ano de nascimento, mostraram variação, sem tendência de alteração e de diferenciação entre os rebanhos no decorrer dos anos. A seleção para peso ao sobreano dentro de rebanho, por 3,5 gerações de seleção, não afetou a permanência das vacas no rebanho até cinco anos de idade. O modelo touro de limiar foi capaz de separar a mudança genética da mudança ambiental.
\end{abstract}

Palavras-chave: bovino de corte, experimento de seleção, modelo de limiar, peso corporal, permanência de vacas no rebanho

\section{Effect of Selection for Growth on the Stayability of Nelore Cows up to Five Years of Age}

\begin{abstract}
Records of 1021 Nelore cows were analyzed in order to evaluate the effect of selection for growth on their stayability in the herd up to five years, considering that they were selected $(\mathrm{P} 5 \mid \mathrm{S})$. The coded variable $(0=$ culled and $1=$ not culled $)$ was analyzed with a threshold model. The Nelore cows were born from 1981 to 1997 within the selected (NeS and NeT) and control (NeC) lines of the Experimental Station of Sertãozinho (SP-Brazil). In NeS and NeT the selected (replacement) animals were those with high selection differentials, within line and year, and in $\mathrm{NeC}$ those with null selection differentials for yearling weight. After 3.5 generations of selection, the NeS and NeT selection weight was $22 \%$ higher compared to NeC. The observed P5|S mean was $68 \%$. Line and selection weight effects were not significant for P5|S. The generation effect was significant, showing a decreasing trend of P5|S along the years in a similar fashion for all the three lines. This tendency could be explained mainly to environmental effects, like increasing replacement rates of heifers, and not to selection for weight. The additive genetic variance, estimated by animal model, sire model and sire and maternal grandsire model were $0.06,0.16$, e, 0.17, proportionally to $\sigma^{2}$ constrained to one, and the heritabilities were $0.06 \pm 0.06,0.15 \pm 0.13$ and $0.16 \pm 0.13$, respectively. The annual average breeding values of sires showed variation, without a regular trend along the years, similarly for the three lines. The selection for yearling weight within-herd, after 3.5 generations, did not cause any change in cow stayability up to five years of age. The threshold sire model was able to separate the genetic from the environmental trend.
\end{abstract}

Key Words: beef cattle, selection experiment, threshold model, body weight, stayability of cows

\section{Introdução}

O peso corporal ou a taxa de crescimento continua sendo o critério de seleção primário para a maioria dos criadores de bovinos de corte em todo o mundo, sendo, portanto, de extrema importância conhecer as consequências da seleção para crescimento sobre outros caracteres de importância econômica para a produção de carne. Há muitas evidências de que a seleção para maior peso corporal em idades jovens

\footnotetext{
1 Instituto de Zootecnia - Estação Experimental de Zootecnia de Sertãozinho - http://www.iz.sp.gov.br/eezooser - Projeto IZ-14006/76.

2 Pesquisador, EEZS-SP, IZ/APTA/SAA-SP. Caixa Postal 63, Cep:14.160-900, Sertãozinho-SP (mezmerca@ig.com.br; razook@iz.sp.gov.br; cyrillo@iz.sp.gov.br; figueiredo@iz.sp.gov.br).

${ }^{3}$ Bolsista do CNPq.
} 
não apresenta efeitos sobre o desempenho reprodutivo das matrizes (Morris et al., 1992; Archer et al., 1998; Mercadante et al., 2000). Entretanto, ainda persiste no Brasil o conceito de que a seleção para crescimento levaria à obtenção de fêmeas com menor eficiência reprodutiva e menor tempo de vida útil.

Um experimento de seleção fornece excelente oportunidade de estudo das relações entre seleção direta e respostas correlacionadas, apesar da limitação em termos do número de animais. Mercadante et al. (2002, 2003) mostraram que, após 3,5 gerações de seleção para maior peso corporal, as matrizes Nelore do Experimento de Seleção de Sertãozinho apresentaram igual desempenho reprodutivo que as selecionadas para peso corporal em torno da média, apesar da grande diferença detectada no peso à seleção. Entretanto, poderia haver diferença na permanência das fêmeas nos rebanhos até certa idade, uma vez que o estudo foi restrito às primeira, segunda e todas as montas juntas.

Hudson \& Van Vleck (1981) usaram a capacidade de permanência no rebanho ("stayability") como uma medida de longevidade, definindo-a como a probabilidade do animal permanecer no rebanho até determinada idade, dada a oportunidade de chegar a esta idade. A capacidade de permanência do animal no rebanho reflete seus desempenhos reprodutivo e produtivo, já que a principal causa de descarte das matrizes é falha reprodutiva, sendo portanto, segundo Snelling et al. (1995), um bom indicador da eficiência da matriz. Predições genéticas para capacidade de permanência das fêmeas no rebanho representam uma oportunidade para se reduzir custos e aumentar lucros pela seleção por persistência de reprodução e produtividade (Snelling et al., 1995; Martínez et al., 2002).

O objetivo deste trabalho foi avaliar o efeito da seleção para peso corporal na permanência da fêmea no rebanho até cinco anos de idade, dado que ela foi selecionada, e estimar a herdabilidade e a mudança genética desta característica em três rebanhos experimentais de bovinos da raça Nelore.

\section{Material e Métodos}

As informações são referentes aos rebanhos da raça Nelore do Experimento de Seleção da Estação Experimental de Zootecnia de Sertãozinho. Os detalhes da implantação dos rebanhos e do processo seletivo foram descritos por Razook et al. (1998). O experimento de seleção iniciou em 1976 e o estabelecimento dos rebanhos Nelore Controle $(\mathrm{NeC})$, Nelore Seleção (NeS) e Nelore Tradicional (NeT) ocorreu na monta de 1980, com respectivamente 60 , 120 e 170 fêmeas e utilizando 4, 6 e até 8 touros/ano. Os machos foram selecionados segundo diferencial de seleção máximo ( $\mathrm{NeS}$ e $\mathrm{NeT})$ e nulo $(\mathrm{NeC})$, dentro de rebanho e ano, do peso padronizado aos 378 dias, obtido após prova de desempenho em confinamento, e as fêmeas, segundo diferencial de seleção máximo ( $\mathrm{NeS}$ e $\mathrm{NeT}$ ) e nulo $(\mathrm{NeC})$ do peso padronizado aos 550 dias (peso à seleção - PS), em regime de pasto, mas, em geral, foram retidas anualmente $50 \%$ delas. $\mathrm{O} \mathrm{NeT}$, em um esquema mais flexível, recebeu touros de outros rebanhos, assim como, eventualmente, alguns touros e matrizes descartados dos $\mathrm{NeC}$ e NeS. As novilhas selecionadas foram submetidas à monta natural aos dois anos de idade e as vacas foram descartadas por falharem dois anos consecutivos, por má habilidade materna ou por idade avançada, por volta dos 12 anos.

A variável resposta foi definida como a permanência da vaca no rebanho até cinco anos de idade, dado que ela foi selecionada (P5|S), e codificada como $0=$ fracasso (descartada) ou $1=$ sucesso (não descartada). Considerando que a informação usada para estabelecer a resposta 0 ou 1 foi a entrada na estação de monta, aos cinco anos as vacas tiveram oportunidade de participar de quatro estações de monta. Foram utilizados os dados coletados entre 1983 a 2002 (5713 registros de 1264 vacas), constituindo uma amostra de 1021 fêmeas com registros de P5|S, nascidas de 1981 a 1997, e filhas de 135 touros. Foram retiradas as vacas que, no momento da análise, não tinham cinco anos (nascidas após 1997).

O coeficiente de geração (CGER) foi calculado como proposto por Brinks et al. (1965), $\mathrm{CG}=$ (CG pai + CG mãe) $/ 2$ + 1, em que CG é o número médio de segregações mendelianas dos pais até os animais fundadores, cujo GC é zero. Considerou-se os animais nascidos em 1981 como pertencentes à primeira geração.

A probabilidade de sucesso para $\mathrm{P} 5 \mid \mathrm{S}$ foi modelada com função de ligação probit (modelo de limiar) em modelo linear generalizado, ajustando alternativamente os efeitos fixos de rebanho, ano de nascimento, PS e CGER com a finalidade de estudar o efeito da seleção na P5|S. O modelo para o peso à seleção (PS) incluiu os efeitos fixos de rebanho, ano de nascimento e a interação rebanho $x$ ano. Para a obtenção do 
componente de variância de P5|S, o modelo ajustado incluiu os efeitos fixos de rebanho e ano de nascimento e o peso à seleção como covariável, assim como o efeito aleatório de animal (modelo animal) ou de touro (modelo touro e modelo touro avô-materno), incorporando matriz de parentesco com 6588 animais.

A função de ligação representa um modelo biológico da média, ou seja, assume-se que as respostas binomiais (0 ou 1) sejam manifestações observáveis de processos subjacentes contínuos não observáveis. Esta idéia de uma variável subjacente normal é compatível com o modelo genético aditivo infinitesimal. O modelo probit, também chamado de modelo de limiar, cuja variância residual na escala subjacente é tomada como uma unidade da medida, $\sigma^{2}=1$ (Gianola \& Foulley, 1983), assegura que as estimativas sejam no intervalo 0 e 1 , isto é, que a média do sucesso de P5|S estimada para cada um dos rebanhos ficará entre 0 e $100 \%$ (Littel et al., 1996). As análises foram feitas utilizando-se osoftware ASREML (Gilmour et al., 1999), que fornece estimativas aproximadas de máxima verossimilhança (quasi-likelihood) para o efeito aleatório no caso de modelo linear generalizado misto. Em casos de sub ou superdispersão dos resíduos, dado pelo parâmetro escala (deviance/graus de liberdade), o ASREML permite utilizar uma $\sigma^{2}{ }_{\mathrm{e}}$ diferente da unidade. As médias ajustadas e as soluções obtidas na escala normal subjacente para $\mathrm{P} 5 \mid \mathrm{S}$, foram re-transformadas aplicando a função de probabilidade da normal acumulada, sendo estas últimas expressas como desvio de $50 \%$, como proposto por Snelling et al. (1994). As herdabilidades foram estimadas respectivamente para modelo animal $\left(\mathrm{h}_{\mathrm{ma}}^{2}\right)$, modelo touro $\left(\mathrm{h}^{2}{ }_{\mathrm{mt}}\right)$ e modelo touro-avô materno $\left(\mathrm{h}^{2}{ }_{\mathrm{mt}-\mathrm{am}}\right)$, como: $h_{\mathrm{ma}}^{2}=\sigma_{\mathrm{a} /}^{2}\left(\sigma^{2}{ }_{\mathrm{a}}+\sigma^{2}{ }_{\mathrm{e}}\right) ; \mathrm{h}^{2}{ }_{\mathrm{mt}}=4 \sigma_{\mathrm{s}}^{2} /\left(\sigma_{\mathrm{s}}^{2}+\sigma^{2}{ }_{\mathrm{e}}\right) \mathrm{e}$, $\mathrm{h}^{2}{ }_{\mathrm{mt}-\mathrm{am}}=4 \sigma_{\mathrm{s}}{ }_{\mathrm{s}} /\left(\sigma_{\mathrm{s}}^{2}+1 / 4 \sigma_{\mathrm{s}}^{2}+\sigma_{\mathrm{e}}^{2}\right)$, em que $\sigma^{2}{ }_{\mathrm{a}}$ é a variância genética aditiva, obtida em modelo animal; $\sigma^{2}$, a variância de touro; e $\sigma^{2}$, a variância residual.

\section{Resultados e Discussão}

A porcentagem de sucesso para $\mathrm{P} 5 \mid \mathrm{S}$ foi de 68 , ou seja, $68 \%$ das fêmeas selecionadas permaneceram pelo menos até cinco anos no rebanho (Figura 1). Snelling et al. (1995) observaram, em vacas Angus, $75 \%$ de sucesso de ter duas crias, dado que a vaca teve uma, e Silva et al. (2003), em vacas Nelore observaram $38 \%$ de sucesso para permanência no rebanho até cinco anos, dado que ela teve, no mínimo, uma cria antes dessa idade. As médias são compará- veis somente se for considerada a porcentagem das fêmeas nascidas que foram colocadas em reprodução. Neste estudo foram submetidas à monta cerca de 50\%, enquanto no de Silva et al. (2003), cerca de 90\%; assim, as médias nos dois estudos são, proporcionalmente, semelhantes. A porcentagem das vacas retidas, relativamente ao número de novilhas colocadas em monta aos dois anos de idade, foi muito semelhante para os rebanhos $\mathrm{NeC}, \mathrm{NeS}$ e $\mathrm{NeT}$ e o maior descarte foi observado entre quatro e cinco anos de idade (Figura 1). Este fato justifica a opção de considerar a permanência da vaca até a entrada da quarta monta, com cinco anos de idade. Van der Westhuizen et al. (2001) mostraram porcentagens bem maiores de descarte de vacas cruzadas, o que pode refletir diferentes decisões de descarte, como também menor capacidade de adaptação das vacas ao ambiente oferecido.

Na Tabela 1, estão as médias ajustadas de PS e P5|S por rebanho e o CGER médio observado, tanto para todas as vacas, como para aquelas nascidas nos últimos quatro anos cujas médias caracterizam melhor a seleção acumulada. As médias do PS das fêmeas de $\mathrm{NeS}$ e $\mathrm{NeT}$ foram cerca de 14 e $22 \%$ acima daquelas do $\mathrm{NeC}$, respectivamente, para todas as vacas e para aquelas nascidas nos últimos quatro anos. Apesar da grande diferença no PS, as médias ajustadas de $\mathrm{P} 5 \mid \mathrm{S}$ foram diferentes somente entre $\mathrm{NeC}$ e $\mathrm{NeT}$ para as vacas nascidas durante todo o

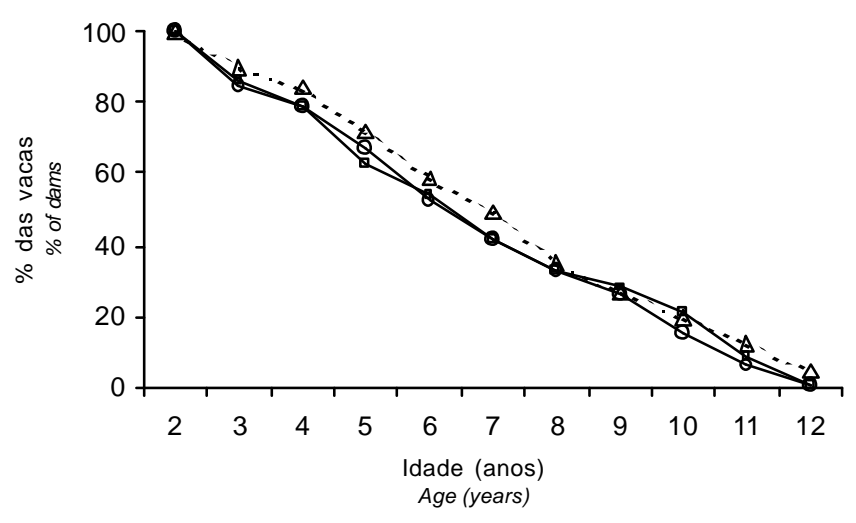

Figura 1 - Porcentagem das vacas de várias idades, relativamente às novilhas colocadas em monta, que foram mantidas no rebanho (controle $\square$, seleção o, tradicional $\Delta$ ).

Figure 1 - Percentage of dams of various ages, relative to the number of selected heifers, that were mantained in the herd (control $\square$, selection $\mathrm{o}$, tradicional $\Delta$ ). 
experimento. Concordando com estes resultados o PS foi não significativo para P5|S, ajustando modelos com e sem o efeito de rebanho. O CGER foi significativo em modelo incluindo o efeito de rebanho, mas com tendência de diminuição da $\mathrm{P} 5 \mid \mathrm{S}$ com o aumento do CGER (-10\%, -15\% e -10\% em NeC, NeS e NeT) nos três rebanhos e não somente nos selecionados, o que indicaria efeito da seleção. O efeito do CGER, juntamente com as médias de P5|S menores para as vacas nascidas nos últimos quatro anos, é explicado pelas mudanças nas práticas de reposição de matrizes ao longo dos anos. Até 1992, submetia-se à monta um número de novilhas igual à necessidade de reposição (20\% das vacas) e, após este ano, optou-se por submeter maior número delas à monta e descartar mais após a primeira ou segunda monta.

As variâncias de origem genética aditiva $\left(\sigma^{2}{ }_{\mathrm{a}}\right)$, estimadas por modelo animal, por modelo touro e por modelo touro avô-materno, foram 0,06; 0,16 e 0,17, proporcionalmente à $\sigma_{\mathrm{e}}^{2}$ fixa em uma unidade da medida e as herdabilidades iguais a 0,06 $\pm 0,06$; $0,15 \pm 0,13$ e $0,16 \pm 13$, respectivamente. Em todas as análises, o parâmetro escala ficou entre 1,15 e 1,18, e as análises considerando a superdispersão forneceram estimativas de herdabilidade fora do espaço paramétrico para modelo animal e iguais a $0,16 \pm 0,13$ e $0,17 \pm 0,13$, respectivamente, para modelo touro e modelo touro avô-materno, com a $\sigma_{\mathrm{e}}^{2}$ próxima da unidade. A estimação de componentes de variância em análise de dados categóricos é extremamente sensível à quantidade de informação. Neste caso, há potencialmente maior quantidade de informação por "unidade experimental estudada" em modelo touro e modelo touro avô-materno que em modelo animal, sendo, portanto, esperada estimativa de $\sigma^{2}$ maior em modelo touro, embora este possa ser considerado menos confiável, já que ignora a informação vinda das mães (Gilmour, 2001). Os erros-padrão das estimativas de herdabilidade foram altos, fazendo com que as estimativas se aproximem de zero e sejam semelhantes para os três modelos, considerando ou não a dispersão dos dados. Gilmour (2001) comenta que os componentes de variância estimados pelo ASREML para dados não normais não são rigorosamente REML, sendo apenas extrapolações deste método. Recomenda-se, então, a utilização de vários modelos, para ter certeza que as conclusões não são modificadas por eles.

Os trabalhos encontrados na literatura relatam herdabilidades para habilidade de permanência no rebanho até cinco ou sete anos, com variações em sua definição, quase sempre obtidas com modelo touro de limiar. Doyle et al. (2000) obtiveram herdabilidade de 0,15 com intervalo de confiança de 0,04 a 0,30 para a habilidade de vacas da raça Angus ter cinco crias dado que ela teve uma cria aos dois

Tabela 1 - Número de animais, coeficiente de geração e médias ajustadas ( \pm erro-padrão) por rebanho para as variáveis peso à seleção e permanência da vaca no rebanho até cinco anos de idade, dado que ela foi selecionada (P5|S)

Table 1 - Number of animals, coefficient of generation and least squares means ( \pm standard error), by line, for selection weight and stayability of dams until five years, considering it was selected (P5/S)

\begin{tabular}{|c|c|c|c|c|}
\hline $\begin{array}{l}\text { Rebanho } \\
\text { Line }\end{array}$ & $\mathrm{N}^{1}$ & $\mathrm{CGER}^{2}$ & $\begin{array}{l}\text { Peso à seleção }(\mathrm{kg}) \\
\text { Selection weight }(\mathrm{kg})\end{array}$ & $\begin{array}{c}\mathrm{P} 5 \mid \mathrm{S} \text { (\% de sucesso) } \\
P 5 \mid S(\% \text { of success })\end{array}$ \\
\hline
\end{tabular}

Todas as vacas All dams

\begin{tabular}{|c|c|c|c|c|}
\hline & & & $P<0,01$ & $P<0,01$ \\
\hline $\mathrm{NeC}$ & 195 & 2,5 & $246 \pm 1^{\mathrm{a}}$ & $65 \pm 4^{\mathrm{a}}$ \\
\hline $\mathrm{NeS}$ & 402 & 2,4 & $280 \pm 1^{b}$ & $70 \pm 2^{\mathrm{ab}}$ \\
\hline $\mathrm{NeT}$ & 424 & 2,2 & $281 \pm 1^{b}$ & $74 \pm 2^{b}$ \\
\hline
\end{tabular}

Vacas nascidas nos últimos quatro anos

Dams born in the last four years

\begin{tabular}{lcccc}
\hline & & $\mathrm{P}<0,01$ & $\mathrm{P}>0,05$ \\
$\mathrm{NeC}$ & 64 & 3,5 & $258 \pm 2^{\mathrm{a}}$ & $55 \pm 6$ \\
$\mathrm{NeS}$ & 134 & 3,5 & $316 \pm 1^{\mathrm{b}}$ & $54 \pm 4$ \\
$\mathrm{NeT}$ & 150 & 3,1 & $308 \pm 1^{\mathrm{b}}$ & $61 \pm 4$ \\
\hline
\end{tabular}

1 Número de animais (Number of animals).

2 Média do coeficiente de geração (Average of coefficient of generation).

a, b Médias na mesma coluna com diferentes letras são significativamente $(P<0,05)$ diferentes.

$a, b$ Means in the same column with different letters are significantly different $(P<.05)$. 
anos de idade e, Martínez et al. (2002), relataram herdabilidade de 0,13 $\pm 0,06$ para habilidade de vacas Hereford permanecerem no rebanho por mais três anos após parirem aos dois anos de idade. Para vacas Nelore, Silva et al. (2003) e Paneto et al. (2002) relataram valores semelhantes $(0,15$ e 0,12 , respectivamente, com intervalo de confiança de 0,07 a 0,17 em ambos) para habilidade de permanência até cinco anos dado que a vaca produziu pelo menos uma cria antes desta idade e habilidade de permanência até seis anos dado que a vaca produziu três crias neste período, respectivamente.

As médias dos valores genéticos dos touros para $\mathrm{P} 5 \mid \mathrm{S}$, apesar de variáveis, mostraram que não houve tendência genética significativa para touros $(0,02 \%)$ ano, 0,06\%/ano e 0,15\%/ano, para $\mathrm{NeC}, \mathrm{NeS}$ e NeT, respectivamente) e que não há diferenças entre os rebanhos selecionados $\mathrm{NeS}$ e $\mathrm{NeT}$ e o $\mathrm{NeC}$ (Figura 2) ao longo dos anos de seleção para peso. A tendência ambiental (Figura 3), obtida das soluções de ano de nascimento com modelo touro e expressa como desvios do ano de 1981, mostra a diminuição de P5|S ao longo dos anos (-2,22\%/ano), concordando com os resultados apresentados na Tabela 1 . Com base ne sses números, observa-se claramente que foi possível separar as mudanças genética e ambiental usando modelo touro de limiar. Nos rebanhos experimentais selecionados e controle de bovinos da raça Hereford,

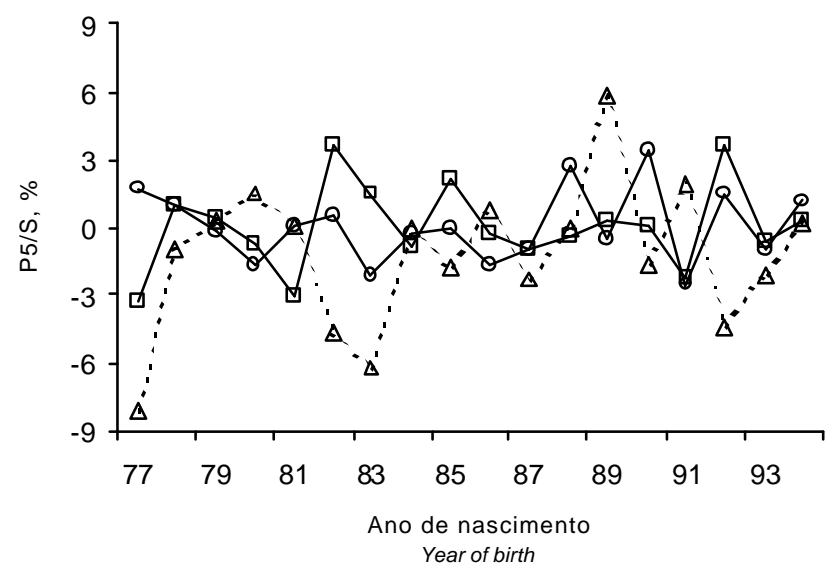

Figura 2 - Média dos valores genéticos, por ano de nascimento, para P5|S dos touros (controle $\square$, seleção o, tradicional $\Delta$ ).

Figure 2 - Annual average breeding values of sires for $P 5 / S$ (controla, selectiono, traditionals). as mudanças genéticas estimadas para permanência de vacas no rebanho por mais três anos após o parto aos dois anos de idade foram também baixas $(0,12 \%)$ ano para o rebanho selecionado para maiores pesos ao desmame, 0,05\%/ano para aquele selecionado para maiores pesos ao sobreano e $0,01 \%$ /ano para o rebanho controle) e a mudança ambiental negativa, igual a -4,04\%/ano (Martínez et al., 2002). Para vacas Nelore, Silva et al. (2003) e Paneto et al. (2002) relataram mudança genética um pouco maior que as observadas em $\mathrm{NeC}$ e $\mathrm{NeS}(0,12 \% / a n o)$ com dados de rebanhos maiores e abertos a material genético de fora.

Os métodos usados para detectar o efeito da seleção para crescimento na $\mathrm{P} 5 \mid \mathrm{S}$ podem ter falhas neste tipo de estrutura de dados, enquanto a comparação de médias omite o relacionamento entre gerações, o que gera implicações importantes na análise de dados gerados por seleção, a análise sob modelo misto de limiar é muito sensível ao número de observações (Gilmour et al., 1999), fornecendo, nesse caso, soluções com alto erro-padrão. Contudo, ambas as análises mostraram que, após 3,5 gerações de seleção para crescimento, a probabilidade de sucesso na $\mathrm{P} 5 \mid \mathrm{S}$ foi semelhante à observada no rebanho "não selecionado", concordando com os resultados obtidos por Mercadante et al. (2002, 2003) para as características de reprodução, dias ao parto e taxa de prenhez.

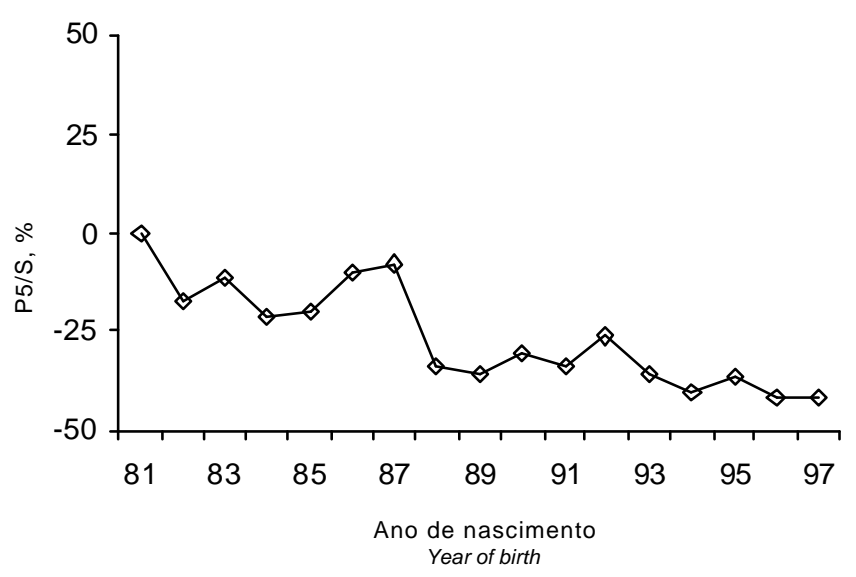

Figura 3 - Mudança ambiental para P5|S (valores expressos como desvios de 1981).

Figure 3 - Environmental trends for P5/S (values expressed as deviation to 1981). 


\section{Conclusões}

A seleção para peso ao sobreano dentro de rebanho, por 3,5 gerações de seleção, não promoveu mudança genética na permanência das vacas no rebanho até cinco anos de idade. O modelo touro de limiar foi capaz de separar a mudança genética da mudança ambiental.

\section{Literatura Citada}

ARCHER, J.A.; ARTHUR, P.F.; PARNELL, P.F. et. al. Effect of divergent selection for yearling growth rate on female reproductive performance in Angus cattle. Livestock Production Science, v.57, p.33-40, 1998.

BRINKS, J.S.; CLARCK, R.T.; KIEFFER, N.M.Evaluation of response to selection and inbreeding in a closed line of Hereford cattle. Washington, Agricultural Research Service. 36p, 1965. (Technical Bulletin, 1323).

DOYLE, S.P.; GOLDEN, B.L.; GREEN, R.D. et al. Additive genetic parameter estimates for heifer pregnancy and subsequent reproduction in Angus females. Journal of Animal Science, v.78, p.2091-2098, 2000.

GIANOLA, D.; FOULLEY, J.L. Sire evaluation for ordered categorical data with threshold model.Genetic, Selection, Evolution, v.15, p.201-224, 1983.

GILMOUR, A.R. http://www.chiswick.anprod.csiro.au/lists/ asreml. 2001.

GILMOUR, A.R.; CULLIS, B.R.; WELHAM, S.J. et. al. ASREML Reference Manual. Orange, Australia: New South Wales Agriculture, 1999. 232p.

HUDSON, G.F.S.; Van VLECK, L.D. Relationship between production and stayability in Holstein cattle. Journal of Dairy Science, v.64, p.2246-2250, 1981.

LITTEL, R.C.; MILLIKEN, G.A.; STROUP, W.W. et al.SAS system for mixed models. Cary: SAS Institute, 1996. 433p.

MARTÍNEZ, G.E.; KOCH, R.M.; CUNDIFF, L.V. et al. Genetic parameters and genetic trends for stayability in Hereford cows. In: WORLD CONGRESS ON GENETICS APPLIED TO LIVESTOCK PRODUCTION, 7., Montpellier, 2002. Proceedings ... CD-ROM. Montpellier, 2002.

MERCADANTE, M.E.Z.; LÔBO, R.B.; OLIVEIRA, H.N. Estimativas de (co)variâncias entre características de reprodução e de crescimento em fêmeas de um rebanho Nelore. Revista Brasileira de Zootecnia, v.29, p.997-1004, 2000.
MERCADANTE; M.E.Z.; PACKER, I.U.; RAZOOK, A.G. et al. Dias ao parto de fêmeas de um experimento de seleção para crescimento. I. Modelo de repetibilidade. Revista Brasileira Zootecnia, v.31, p.1715-1725, 2002.

MERCADANTE; M.E.Z.; PACKER, I.U.; RAZOOK, A.G. et al. Direct and correlated responses to selection for yearling weight on reproductive performance of Nelore cows. Journal of Animal Science, v.81, p.376-384, 2003.

MORRIS, C.A.; BAKER, R.L.; HUNTER, J.C. Correlated responses to selection for yearling or 18-month weight in Angus and Hereford cattle.Livestock Production Science, v.30, p.33-52, 1992.

PANETO, J.C.C., SILVA, J.A.V.; BEZERRA, L.A.F. et al. Expected response to selection on stayability and its economic weight in a population of Nelore cattle in Brazil. In: WORLD CONGRESS ON GENETICS APPLIED TO LIVESTOCK PRODUCTION, 7., Montpellier, 2002. Proceedings... CD-ROOM. Montpellier, 2002.

RAZOOK, A.G.; FIGUEIREDO, L.A.; BONILHA NETO, L.M. et al. Selection for yearling weight in Nelore and Guzera zebu breeds: selection applied and response in 15 years of progeny. In: WORLD CONGRESS ON GENETICS APPLIED TO LIVESTOCK PRODUCTION, 6., 1998, Armidale. Proceedings... Armidale, 1998. p.133-136.

SILVA, J.A. II V.; ELER, J.P.; FERRAZ, J.B.S. et al. Heritability estimate for stayability in Nelore cows. Livestock Production Science, v.79, p.97-101, 2003.

SNELLING, W.M.; GOLDEN, B.L.; BOURDON, R.M. An EPD for stayability of beef cows. In: WORLD CONGRESS ON GENETICS APPLIED TO LIVESTOCK PRODUCTION, 5., 1994, Guelph.Proceedings... Guelph, 1994. p.169-172.

SNELLING, W.M.; GOLDEN, B.L.; BOURDON, R.M. Withinherd genetic analyses of stayability of beef cattle. Journal of Animal Science, v.73, p.993-1001, 1995.

Van der WESTHUIZEN, R.R.; SCHOEMAN, S.J.; JORDAAN, G.F. et al. Heritability estimates derived from threshold analyses for reproduction and stayability traits in a beef cattle herd. South African of Animal Science, v.31, p.25-32, 2001.
Recebido em: 18/11/02 Aceito em: 11/07/03 